\title{
Incipient oxidation of magnesium: A high-resolution electron-energy-loss and photoemission study
}

\author{
P. A. Thiry \\ Laboratoire Interdisciplinaire de Spectroscopie Electronique, Institut de Recherches sur les Interfaces Solides, \\ Facultés Universitaires Notre-Dame de la Paix, 61 rue de Bruxelles, B-5000 Namur, Belgium \\ J. Ghijsen* \\ Max-Planck-Institut für Festkörperforschung, Heisenbergstrasse 1, Postfach 8006 65, D-7000 Stuttgart 80, \\ Federal Republic of Germany \\ R. Sporken and J. J. Pireaux \\ Laboratoire Interdisciplinaire de Spectroscopie Electronique, Institut de Recherches sur les Interfaces Solides, \\ Facultés Universitaires Notre-Dame de la Paix, 61 rue de Bruxelles, B-5000 Namur, Belgium \\ R. L. Johnson \\ Max-Planck-Institut für Festkörperforschung, Heisenbergstrasse 1, Postfach 8006 65, D-7000 Stuttgart 80, \\ Federal Republic of Germany \\ R. Caudano \\ Laboratoire Interdisciplinaire de Spectroscopie Electronique, Institut de Recherches sur les Interfaces Solides, \\ Facultés Universitaires Notre-Dame de la Paix, 61 rue de Bruxelles, B-5000 Namur, Belgium
}

(Received 11 July 1988)

\begin{abstract}
The initial stages of magnesium oxidation are investigated by high-resolution electron-energy-loss spectroscopy. Two species (magnesium oxide and chemisorbed oxygen) are simultaneously detected from their vibrational bands even for minimal oxygen exposures. The evolution of the surface oxide can be quantitatively described by dielectric theory using infrared optical constants deduced from measurements on $\mathrm{MgO}$ single crystals. The proposed oxidation model is confirmed by the $\mathrm{Mg} 2 p$ electron-distribution curves obtained with synchrotron radiation.
\end{abstract}

\section{INTRODUCTION}

There is considerable technological as well as fundamental scientific interest in the study of the oxidation of metal surfaces. Particularly important is the observation of the early stages of interaction when the reacting gas is brought into contact with the clean metallic surface. The details of these first steps often condition the oxidation process and must be known to understand the exact oxidation mechanism. Magnesium is an example of a metal whose incipient surface oxidation has been little studied experimentally. The reason probably lies in the difficulties encountered in sample preparation because of its high reactivity with oxygen. Most previous studies have concentrated on evaporated films and, up to now, very few experiments on single-crystal surfaces have been reported, although they should give more precise information because low-energy electron diffraction (LEED) measurements are now feasible.

Namba et al. ${ }^{1}$ proposed a four-stage model for the incipient oxidation of $\mathrm{Mg}(0001)$ based on LEED, Augerelectron spectroscopy (AES), electron energy-loss spectroscopy (EELS), and work-function measurements: (1) random oxygen chemisorption followed by oxygen incorporation, (2) assembly into islands and lateral growth, (3) oxide formation, (4) oxide thickening. They emphasized the influence of surface roughness on their results. The adsorption of oxygen on $\mathrm{Mg}(0001)$ was studied by Ghijsen et al. ${ }^{2}$ using $\mathrm{x}$-ray photoemission spectroscopy (XPS). After the immediate formation of oxide they also observed another oxygen species (defective oxide or chemisorbed oxygen). Hayden et al. ${ }^{3}$ investigated the early stages of oxidation of $\operatorname{Mg}(0001)$ and $\operatorname{Mg}(1 \overline{1} 00$ ) single-crystal surfaces by LEED, EELS, work function, and ellipsometric measurements. They reported the formation of an incorporated $(1 \times 1)$ oxygen underlayer up to monolayer coverage (about $6 \mathrm{~L}$ exposure, where $\mathrm{L}$ denotes langmuirs, or $10^{-6}$ Torrs) and its subsequent transformation into epitaxial oxide.

Flodström and Martinsson ${ }^{4}$ studied the initial oxidation of $\mathrm{Mg}(0001)$ by AES, LEED, and EELS. They identified three successive stages: (1) oxygen chemisorption below the top $\mathrm{Mg}$ layer, (2) formation of an oxide layer, and (3) oxide thickening. They also presented some evidence for epitaxial oxide formation.

From this summarized review of the literature, it appears that there is a discrepancy between the XPS and the results from other techniques. Although XPS reports immediate oxide formation, all other authors observe a "precursor" chemisorption stage, before oxide formation. The purpose of this paper is to address this issue in the early oxidation of $\mathrm{Mg}$ single-crystal surfaces. $\mathrm{As}$ it is necessary to use highly surface-sensitive techniques, we have chosen high-resolution electron-energy-loss spec- 
troscopy (HREELS) and synchrotron-radiation-induced photoemission spectroscopy whose capabilities for surface and interface studies have been abundantly demonstrated in recent years.

It has been shown on several occasions that HREEL spectra of oxides and semiconductors can be quantitatively explained by the dielectric theory. This is the reason why we will start this study with a detailed description of the HREELS results obtained on $\mathrm{MgO}$ single crystals. These results will serve as a reference to characterize the two oxygen species observed during oxygen exposure of the $\mathrm{Mg}$ surfaces. An attempt will be made to extend the use of the dielectric theory in order to interpret quantitatively the HREEL spectra recorded during Mg oxidation. We succeed for one oxygen species by invoking an empirical rule valid for diatomic gas molecules and whose application we believe can be relevant in the solid-state context. The other oxygen species will be only qualitatively discussed.

The resulting oxidation model is that of direct incorporation of atomic oxygen in the magnesium, leading to the simultaneous formation of $\mathrm{Mg}$ oxide and of a lessbonded oxygen chemisorbed at the surface. This model is confirmed by the analysis of the $\mathrm{Mg} 2 p$ electrondistribution curves (EDC's) observed by photoemission using synchrotron radiation.

\section{HREELS EXPERIMENTAL SETUP}

The HREELS measurements were taken with a singlepass $180^{\circ}$ hemispherical electron spectrometer (SEDRA, I.S.A. Riber) consisting of a monochromator and an analyzer. All spectra were recorded in the specular scattering geometry with an angle of incidence of $45^{\circ}$. The electron impact energy was chosen so as to provide maximum electronic reflectivity. The exact electron energy was measured in the case of $\mathrm{MgO}$ by scanning the HREEL spectrum up to the cutoff of the intensity. For the metallic samples the electron beam energy is determined from the accelerating potential of the last electrode, i.e., the reported values are not corrected for work-function differences between sample and emitting cathode.

\section{MAGNESIUM OXIDE}

\section{A. Sample preparation}

Two kinds of magnesium-oxide single crystals from Spicer Ltd. were used. They had $99.999 \%$ and $99.9 \%$ purity, respectively. The samples were cleaved in air just before their loading in the ultrahigh-vacuum (UHV) system. Cleavage produced well-oriented (001) surfaces, with very few visible steps or defects. LEED patterns showed no reconstruction and, after a brief heat treatment at $900 \mathrm{~K}$, no contamination could be detected by AES using a retarding-field analyzer.

The 99.9\%-pure samples were conducting enough to be analyzed by HREELS without problems due to charging effects; however, the spectra were of poor quality. In order to stabilize the surface potential on the insulating 99.999\%-pure samples, a special experimental setup was necessary. This setup uses the defocused beam of an Auger-electron gun to "flood" the surface with highenergy electrons. Details about the operation of this gun and about the explanation of its neutralization effects have been extensively discussed elsewhere. ${ }^{5}$ For the measurements reported here the settings of this auxiliary electron gun were typically $2.8-\mathrm{keV}$ energy and less than $1 \mu \mathrm{A}$ intensity.

\section{B. Results and interpretation}

The HREELS measurements on MgO single crystals were intended to serve as a reference for characterization of the oxide formation during exposure of magnesium to oxygen. In this framework, they will also be used here to establish the dielectric theory which will be tentatively applied in the following section to describe the oxidation process.

The experimental spectrum of Fig. 1 has been recorded with the use of the neutralization electron gun. From both sides of the elastic peak, it displays a series of peaks equally spaced by $651 \mathrm{~cm}^{-1}$ and related to the excitation or annihilation of Fuchs-Kliewer optical surface phonons. ${ }^{6}$ These phonons are two-dimensional waves traveling parallel to the surface. They are surface waves because their amplitudes decay exponentially in the $z$ direction perpendicular to the surface.

Solving Maxwell's equations in the vacuum and the bulk $\mathrm{MgO}$ while taking into account the proper boundary conditions at the surface yields the result that the decay length of a Fuch-Kliewer phonon is equal to the inverse of the modulus of its two-dimensional wave vector. In the present experimental conditions (low electron impact energy and small analyzer aperture $\psi_{c}$ ) the electrons entering the detector will have experienced very small changes of their wave-vector component parallel to the surface. Calculations show that the maximum wave vector transferred to the phonon is $k_{\max }=k_{0} \psi_{c}, k_{0}$ being the deBroglie wave vector of the incident electron. ${ }^{7} \mathrm{~A}$ value

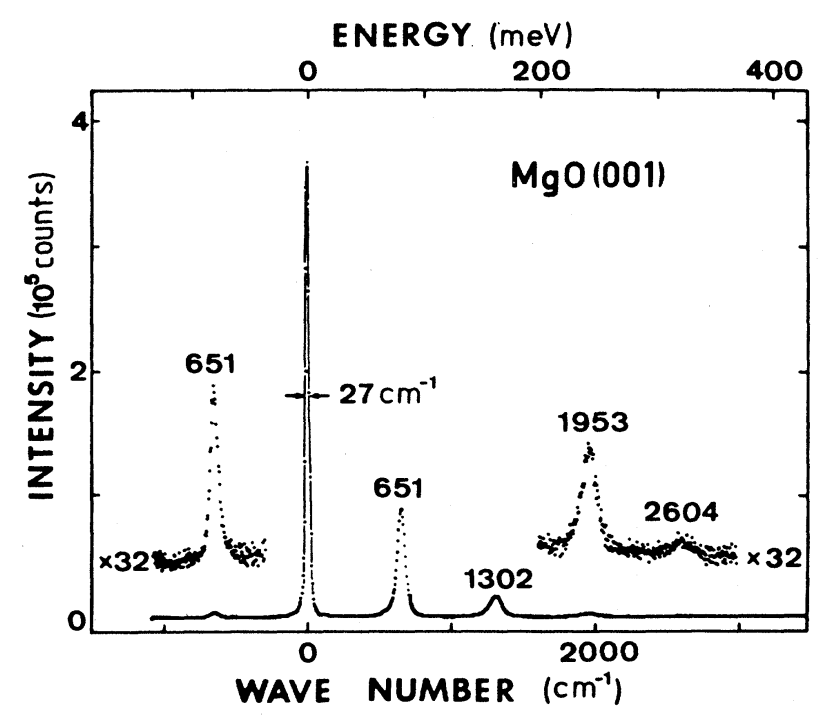

FIG. 1. HREEL spectrum of 11.6-eV electrons specularly reflected from $\mathrm{MgO}(001)$. The angle of incidence is $45^{\circ}$. 
of $0.04 \AA^{-1}$ is obtained for $k_{\max }$ under our experimental conditions, which means that the range of the observed phonons is of the order of $10^{2} \AA$. This long-range property of the Fuchs-Kliewer modes is very important because it allows nondestructive investigations of interfaces of dielectric materials by HREELS. This has been recently reported in two cases: the $\mathrm{CaF}_{2} / \mathrm{Si}(111)$ interface ${ }^{8}$ and a compound semiconductor superlattice GaAs$\mathrm{Al}_{x} \mathrm{Ga}_{1-x}$ As. $^{9}$ Another consequence of the longwavelength character of the Fuchs-Kliewer phonons is that they will be less sensitive to the detailed microscopic structure of the surface and they will allow the use of continuum theories such as the dielectric theory. ${ }^{10}$

\section{Frequency dependence}

A complete derivation of the semiclassical dielectric theory of electron energy loss has been presented in several papers listed in the references, ${ }^{11}$ so that we can start here with the general result obtained in Ref. 7 for the "classical energy-loss probability" $P_{\mathrm{cl}}$ describing the single event corresponding to the excitation of one surface mode:

$$
\begin{aligned}
P_{\mathrm{cl}}(\omega)=\frac{4}{\pi^{2}} \frac{e^{2}}{\hbar v_{\perp}} \int_{D} & d^{2} q \frac{1}{q^{2}} \frac{\left(q v_{\perp}\right)^{3}}{\left[\left(\omega-\mathbf{q} \cdot \mathbf{v}_{\|}\right)^{2}+\left(q v_{\perp}\right)^{2}\right]^{2}} \\
& \times \operatorname{Im} \frac{-1}{\xi_{0}(\mathbf{q}, \omega)+1} .
\end{aligned}
$$

$\xi_{0}(\mathbf{q}, \omega)$ can be considered as an "effective" dielectric function. For a semi-infinite isotropic medium such as the $\mathrm{MgO}$ fcc lattice, $\xi_{0}(\mathbf{q}, \omega)$ reduces to the well-known frequency-dependent dielectric function $\epsilon(\omega)$ which can be removed from the integral. In this case, $P_{\mathrm{cl}}(\omega)$ factorizes as the product of two functions.

(i) The integral over a domain $D$ delimited by the angular acceptance of the analyzer. It gives an amplitude function depending strongly on the kinematic parameters: phonon wave vector $\mathbf{q}$ and electron velocity components $\left(v_{\perp}, \mathbf{v}_{\|}\right)$. This function has a lobed spatial structure that peaks sharply very close to the specular reflected beam. It is known as the "dipole lobe" and has an angular width approximately equal to $\hbar \omega / 2 E_{0}, \hbar \omega$ and $E_{0}$ being the phonon and electron energies, respectively. ${ }^{12}$

(ii) A shape function $\operatorname{Im}\{-1 /[\epsilon(\omega)+1]\}$ depending only on the infrared optical constants of the material through the classical Lorentzian model of the dielectric function $\epsilon(\omega)$ in the phonon range:

$$
\epsilon(\omega)=\epsilon_{\infty}+\frac{\left(\epsilon_{0}-\epsilon_{\infty}\right) \omega_{\mathrm{TO}}^{2}}{\omega_{\mathrm{TO}}^{2}-\omega^{2}-i \gamma \omega \omega_{\mathrm{TO}}} .
$$

$\epsilon_{0}$ and $\epsilon_{\infty}$ are the static and high-frequency dielectric constants, respectively. $\omega_{\text {TO }}$ is the resonance frequency and $\gamma$ is its damping ratio. These infrared optical constants are usually known from infrared reflection spectroscopy (IRS) measurements so that a theoretical classical loss probability can be calculated $a$ priori and, after a special folding ("Poissonization") followed by the instrumental broadening, ${ }^{13}$ can be compared to the experimen- tal HREEL spectrum. This provides a convenient way of determining infrared optical constants by fitting the electron energy-loss data in a similar way as it is done in infrared reflectivity, when a classical dispersion analysis is applied.

The HREEL spectra of $\mathrm{MgO}$ are best fitted with the following optical constants:

$$
\omega_{\mathrm{TO}}=393 \mathrm{~cm}^{-1}, \quad \epsilon_{0}-\epsilon_{\infty}=6.85, \quad \gamma=0.089 .
$$

These values should be compared to the equivalent room-temperature optical constants of $\mathrm{MgO}$ calculated from infrared reflection spectra by Piriou ${ }^{14}$ and Jasperse et al. ${ }^{15}$ which are listed in Table I. As can be seen, they needed two oscillators to fit their reflectivity curves. The most important one, at about $400 \mathrm{~cm}^{-1}$, corresponds to the single resonance frequency deduced by HREELS. The second oscillator, which has a low strength and is heavily damped, is located at a frequency of $640 \mathrm{~cm}^{-1}$, close to the Fuchs-Kliewer phonon. As IRS is not sensitive to surface modes, this second oscillator cannot be attributed to a coupling of light with Fuchs-Kliewer phonons, but it is usually interpreted as arising from anharmonicity effects (combined excitation of bulk acoustic phonons). It is thus coincidental that this frequency matches almost exactly the frequency corresponding to the energy-loss peak observed by HREELS.

Furthermore, two-oscillator behavior is also observed on alkali halides such as LiF (Refs. 15 and 16) or NaF (Ref. 17), but in that case there is no overlap with the Fuchs-Kliewer frequency measured by HREELS. It is also clear that a second oscillator could be added in the HREELS calculations in order to account for the much higher damping of $35 \mathrm{~cm}^{-1}$. But higher damping values are a common feature of HREELS (Ref. 18), so that it is not necessary to introduce a second oscillator, which is also not evident from the HREEL spectra.

Let us emphasize that an accurate determination of the infrared optical constants of $\mathrm{MgO}$ was only possible due to the excellent energy resolution of the measurements. Such an energy resolution which is evaluated from the width of the elastic peak $\left(27 \mathrm{~cm}^{-1}\right)$, is more easily obtained on insulators because of the absence of low-energy excitations such as plasmons or electron-hole-pair creation.

So far, only the frequency dependence of the classical loss probability was checked by the dielectric theory in

TABLE I. Infrared optical constants of MgO determined at room temperature by HREELS (this work) and by infrared reflection spectroscopy (IRS) measurements $\left(\epsilon_{\infty}=3.01\right)$.

\begin{tabular}{lccc}
\hline \hline & $\begin{array}{c}\omega_{\text {To }} \\
\left(\mathrm{cm}^{-1}\right)\end{array}$ & $\Delta \epsilon$ & $\gamma$ \\
\hline HREELS & $393 \pm 3$ & $6.85 \pm 0.1$ & $0.089 \pm 0.01$ \\
IRS $^{\mathrm{a}}$ & $401 \pm 1$ & $6.60 \pm 0.05$ & $0.0190 \pm 0.006$ \\
& $640 \pm 3$ & $0.045 \pm 0.005$ & $0.160 \pm 0.010$ \\
IRS $^{\mathrm{b}}$ & $397.5 \pm 1$ & $7.30 \pm 0.5$ & $0.0136 \pm 0.0005$ \\
& $643 \pm 1$ & $0.012 \pm 0.003$ & $0.053 \pm 0.008$ \\
\hline \hline
\end{tabular}

${ }^{\mathrm{a}}$ Reference 15 .

${ }^{\mathrm{b}}$ Reference 14 . 
the HREELS-IRS comparison. We have also performed two sets of measurements, in order to check the impactenergy dependence and the angle of incidence dependence of the HREELS cross sections.

\section{Impact-energy dependence}

In this case integrated intensities of the first energy-loss peak $\left(I_{1}\right)$ divided by the elastic peak intensity $\left(I_{0}\right)$ are the relevant quantities, as these ratios directly compare to the frequency integrated classical loss probability $\boldsymbol{P}_{\mathrm{cl}}$. When the electron impact energy is increased, the dipole lobe tends to shrink so that it becomes completely subtended by the detector. Consequently, one may carry out the integration in Eq. (1) over the total solid angle of $4 \pi$ without loss of accuracy and get the following asymptotic behavior:

$$
I_{1} / I_{0}=K v_{1}=K^{\prime} E_{0}^{-1 / 2}
$$

$K$ and $K^{\prime}$ being constants. This characteristic energy dependence of $E_{0}^{-1 / 2}$ at high electron energies was confirmed by Ibach on ZnO (Ref. 19) early in the development of HREELS and was considered at that time as an important test of the dielectric theory proposed by Lucas and Šujić. ${ }^{10}$ We have performed similar measurements on $\mathrm{MgO}$ and the results are indicated in Fig. 2 on a log-log plot where the theoretical line reproduces an "exact" calculation based on formulas given in Ref. 20. The decrease of the cross section at low electron energies is caused by the broadening of the dipole lobe which opens up and whose angular aperture becomes larger than that of the analyzer.

\section{Angle of incidence dependence}

Figure 3 shows a collection of HREEL spectra recorded in specular geometry on $\mathrm{MgO}$ with the same electron impact energy of $6.1 \mathrm{eV}$, for different angles of incidence varying from $40^{\circ}$ to $80^{\circ}$. The intensity of each spectrum

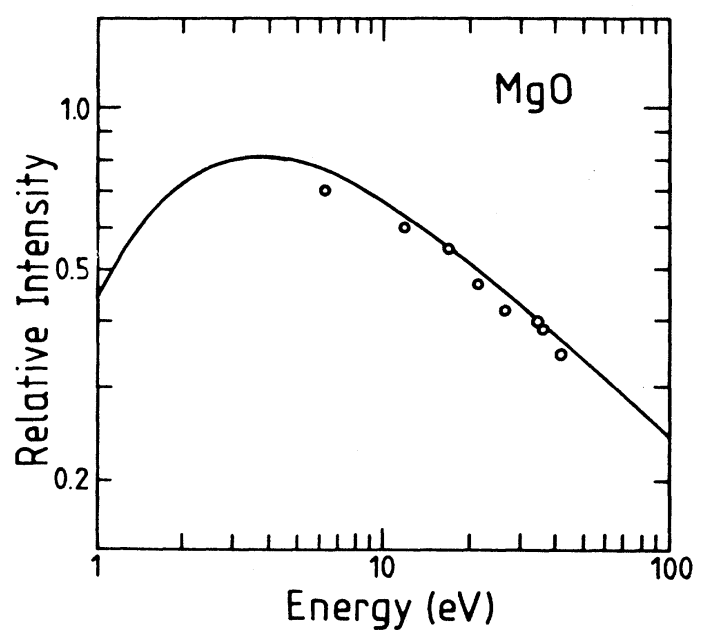

FIG. 2. Relative intensity $\left(I / I_{\text {elastic }}\right)$ of the first-order energy-loss peak measured by HREELS on MgO(001). The solid line is the result of a calculation from the dielectric theory (see text and Ref. 20).

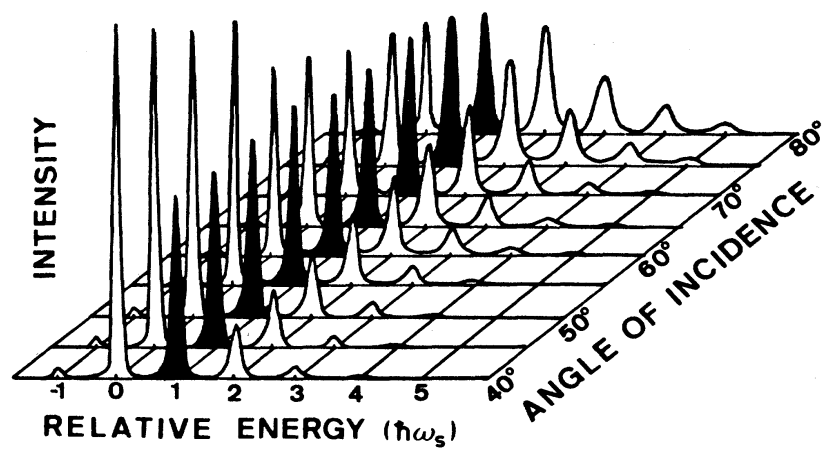

FIG. 3. Collection of energy-loss spectra of 6.1-eV electrons after specular reflection on a $\mathrm{MgO}(001)$ surface. The angle of incidence is varied from $40^{\circ}$ to $80^{\circ}$, in steps of $5^{\circ}$. All spectra are normalized by keeping constant the number of electrons counted.

has been normalized so that the integral (i.e., the total number of electrons counted) is constant for all spectra. One can see the evolution of the classical loss probability, which can be roughly evaluated from the intensity ratios of the first energy-loss peak (in black in Fig. 3) and the elastic peak.

The angular cross sections have been analyzed in detail in two papers by Thiry et al. ${ }^{6}$ and Lambin et al. ${ }^{20}$ In the former paper, a rather crude version of the dielectric theory was used where the wave-vector integral in Eq. (1) was performed approximately. Nevertheless, satisfactory agreement could be obtained with the measured data, by introducing an "effective" analyzer aperture of $1.8^{\circ}$ instead of the geometrical value of $1.2^{\circ}$ and by taking into account image-charge effects. This calculation has been repeated recently with more accuracy by Lambin et al. ${ }^{20}$ using an exact formalism of dielectric theory. They reproduced the measured cross sections to a high level of accuracy.

To conclude this section, we point out the excellent quantitative agreement found in the interpretation of HREELS data of $\mathrm{MgO}$ by the semiclassical dielectric theory, when the following conditions are fulfilled: ${ }^{21}$ (1) correct choice of impact energy, (2) smooth reflectivity in the impact-energy range, (3) avoidance of grazing angles of incidence, and (4) detection in the specular reflected beam. In the following sections, we will attempt to apply the same formalism to the description of the oxidation of Mg single-crystal surfaces.

\section{OXIDATION OF MAGNESIUM}

\section{A. Sample preparation}

The samples used for the oxidation studies were magnesium single crystals $(99.99 \%$ purity) from Metal Crystals and Oxides Ltd. They were oriented with a precision better than $1^{\circ}$ to $\mathrm{Mg}(0001)$ which is the hexagonal basal plane, and $\mathrm{Mg}(1 \overline{100})$ which is the orientation of a lateral face of the hexagonal prism constituting the $\mathrm{Mg}$ elementary cell. After mechanical polishing, they were electrochemically polished in a solution containing orthophos- 
phoric acid, ethanol, and water until a bright mirrorlike surface was obtained. In situ cleaning was performed by repeated cycles of Ar-ion bombardment $(500 \mathrm{eV}, 2$ $\mu \mathrm{A} / \mathrm{cm}^{2}$ at $45^{\circ}$ of incidence, $\left.1 \mathrm{~h}\right)$ and annealing at a temperature not higher than $440 \mathrm{~K}$ in order to prevent magnesium evaporation. ${ }^{1}$ After this cleaning treatment, sharp primitive LEED patterns were observed at $50 \mathrm{eV}$ in accordance with the crystallographic hexagonal-closedpacked structure and no contaminants could be detected by AES. However, it is clear that the resulting surface topography is far from being perfect due to the roughness caused by the ion sputtering. This should be borne in mind during the discussion of the experimental results.

At this point, the sample was introduced in the analysis chamber and the cleaning was further monitored by HREELS. Brief sputter-anneal cycles were then repeated until the closest approximation was reached to an "ideal" surface, i.e., a surface for which only an elastic peak and no energy-loss peak was observed. The cleaning treatment was stopped when no further improvement of the surface quality could be detected. Figure 4 shows the best clean $\mathrm{Mg}$ surface ever obtained before the oxidation experiments. One can still detect some traces in the frequency region where the oxygen vibrations will appear. The oxygen concentration on the surface was estimated to be 0.01 monolayer in this case.

Research-grade oxygen $(99.998 \%$ purity) was introduced in the analysis chamber through a leak valve, after passing through a dry-ice-cooled trap in order to reduce the water contamination. The background pressure in the analysis chamber was $8 \times 10^{-9} \mathrm{~Pa}\left(5 \times 10^{-11}\right.$ Torr $)$. Oxygen pressures of $6.7 \times 10^{-7} \mathrm{~Pa}$ have been used up to 1 $\mathrm{L}$ exposure; $1.33 \times 10^{-6}$ up to $5 \mathrm{~L} ; 6.7 \times 10^{-6} \mathrm{~Pa}$ up to 20 L; higher exposures were performed in the preparation chamber with $1.33 \times 10^{-4}$ and $6.7 \times 10^{-4} \mathrm{~Pa}$. The pressures indicated in the text are uncorrected gauge read-

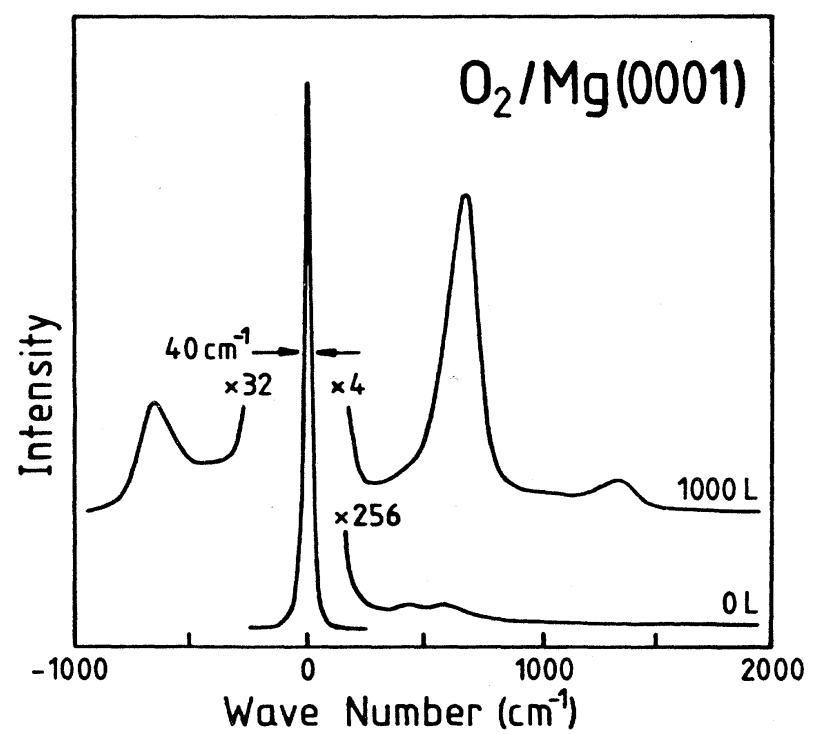

FIG. 4. Energy-loss spectrum of $6.5 \mathrm{eV}$ electrons reflected on a clean $\mathrm{Mg}(0001)$ surface and after adsorption of oxygen (1000 L). The residual oxygen contamination visible on the lower trace is estimated to be of the order of 0.01 monolayers. ings. During the oxygen exposures in the analysis chamber, the HREELS filament was switched off.

\section{B. HREELS results (Ref. 22)}

Figure 5 summarizes a typical sequence of HREEL spectra recorded during the early oxidation process of $\mathrm{Mg}(0001)$. As already mentioned, a slight oxide contamination is observed before gas introduction: in this particular case it corresponds to a coverage of about $4 \%$ of a monolayer $\left(1\right.$ monolayer $=1.12 \times 10^{15}$ atom $\left./ \mathrm{cm}^{2}\right)$. From the start, two vibrational bands are observed at 460 and $620 \mathrm{~cm}^{-1}$. These two peaks, which will be labeled $P 1$ and $P 2$, are relatively broad and $P 1$ appears as a shoulder except in the spectrum at $2 \mathrm{~L}$ coverage, where it is clearly resolved. For exposures higher than $10 \mathrm{~L}$, one observes a third vibrational band on Fig. 4 at $3700 \mathrm{~cm}^{-1}$ and two other structures at 1130 and $1290 \mathrm{~cm}^{-1}$ which can be attributed to combination bands. Above $100 \mathrm{~L}$, additional energy-gain peaks appear which correspond to the first two vibrational bands. Similar spectra were recorded on Mg(1̄̄00).

Peak positions, widths, and intensities were derived by fitting mixed Gaussian-Lorentzian curves to the measured data after subtraction of an exponential background corresponding to the right wing of the elastic

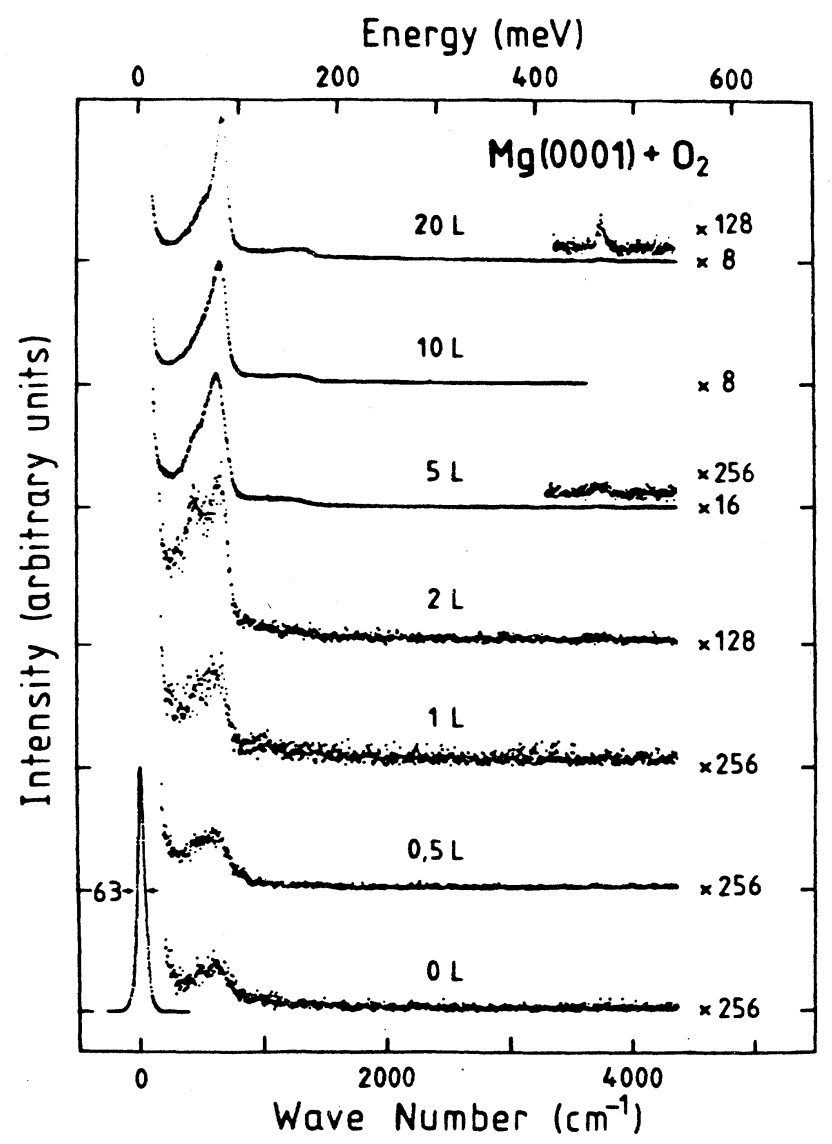

FIG. 5. HREEL spectra of 6.5-eV electrons recorded during early exposure of $\mathrm{Mg}(0001)$ to oxygen $(0.5-20 \mathrm{~L})$. The angles of incidence and of analysis are $45^{\circ}$. 
peak. The intrinsic Lorentzian peak profile is convolved with the instrument transfer function, which in our case is an asymmetric Gaussian. Figure 6 shows that the positions of the two peaks $P 1$ and $P 2$ shift to high frequencies after passing through local minima at about $4 \mathrm{~L}$ for $\mathrm{Mg}$ (0001) and $2 \mathrm{~L}$ for $\mathrm{Mg}(1 \overline{1} 00)$.

The intensities measured with respect to the elastic peak yield the classical loss probability, but a precise determination is difficult because the intensities depend strongly on the shape of the background. Different fits can give errors up to $20 \%$ and the intensities vary between cycles of oxygen exposure, however, the qualitative behavior is the same in all cases. A characteristic series of relative intensities is shown in Fig. 7 for $\mathrm{Mg}(0001)$. We designate the relative intensities of $P 1$ and $P 2$ as $I_{1} / I_{0}$ and $I_{2} / I_{0}$. One observes a regular increase of $I_{2} / I_{0}$, with an inflection point at about $4 \mathrm{~L}$ and saturation after $200 \mathrm{~L}$. The relative intensity $I_{1} / I_{0}$ of $P 1$ follows that of $P 2$ at low exposure, reaches a maximum at about $20 \mathrm{~L}$ and then levels off rapidly.

At low exposure $(<5 \mathrm{~L})$, the widths of $P 1$ and $P 2$ are very similar, but at high coverages $P 1$ becomes substantially broader than $P 2$. Typically, for an energy resolution of $70 \mathrm{~cm}^{-1}$ [full width at half maximum (FWHM) of the elastic peak] at $10 \mathrm{~L}, P 1$ will have a width of 240 $\mathrm{cm}^{-1}$ and $P 2120 \mathrm{~cm}^{-1}$.

Figure 8 shows that the elastic-peak intensities with respect to oxygen exposure are completely different for the two surfaces (0001) and (1100). On $\mathbf{M g}(1 \overline{1} 00)$ the intensity decreases with coverage, but on $\mathrm{Mg}(0001)$ there is an increase. No work-function compensation was applied to make these measurements, ${ }^{23}$ but the HREELS filament was allowed to recover up to the initial monochromator current value. These results will be discussed in a following section.

\section{The dielectric theory}

Before we discuss the HREELS results, it is interesting to apply dielectric theory to predict the effects observed during the early oxidation of magnesium. The problem amounts to constructing a suitable "effective dielectric

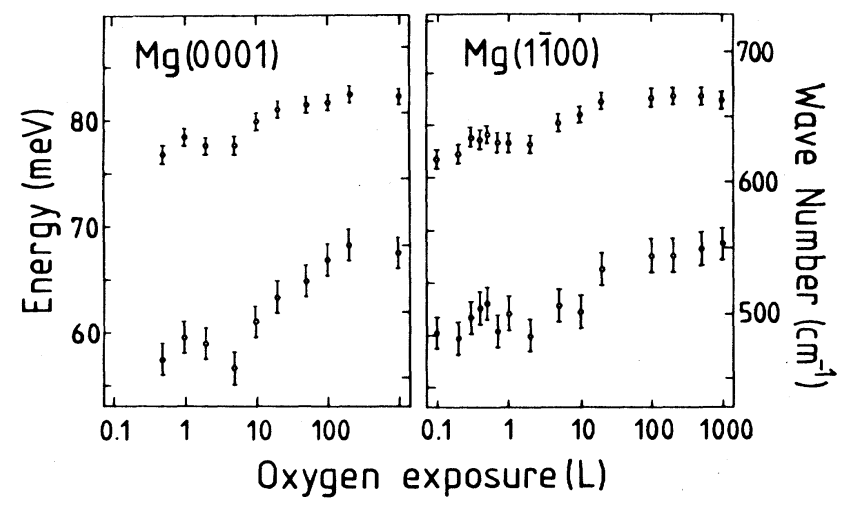

FIG. 6. Evolution of the frequencies of the peaks $P 1$ (chemisorbed oxygen) and $P 2$ (surface oxide) observed during exposure of $\mathrm{Mg}(0001)$ and $\mathrm{Mg}(1 \overline{1} 00)$ to oxygen. One observes a local minimum at about $4 \mathrm{~L}$ on $\mathrm{Mg}(0001)$ and at $2 \mathrm{~L}$ on $\mathrm{Mg}(1 \overline{1} 00)$.

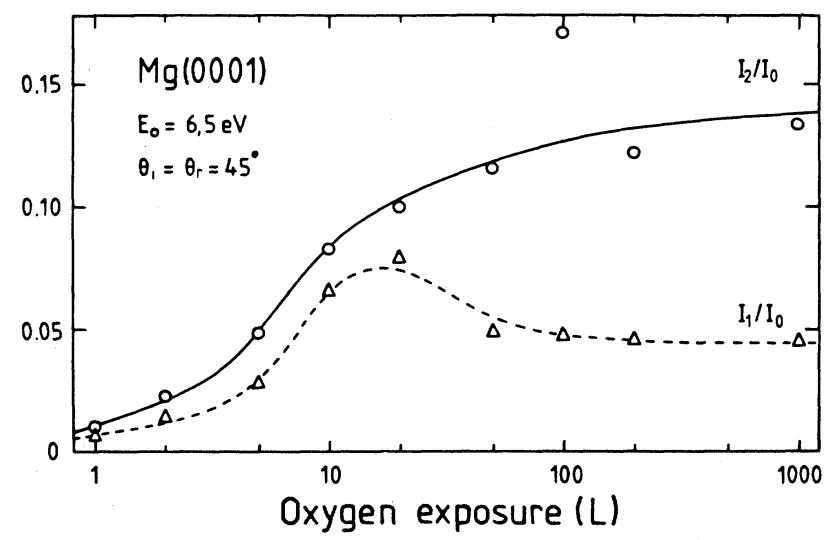

FIG. 7. Relative intensities $\left(I_{1} / I_{\text {elastic }}\right.$ and $\left.I_{2} / I_{\text {elastic }}\right)$ of the two peaks $P 1$ and $P 2$ observed on $\mathrm{Mg}(0001)$ as a function of oxygen exposure. The error bars are at least $20 \%$ of the experimental data. The solid lines are only guides to the eye.

function" $\xi_{0}(\mathbf{q}, \omega)$ to be introduced in the classical energy-loss probability.

For a single layer of thickness $d$ with dielectric function $\epsilon_{1}(\omega)$, supported by a semi-infinite homogeneous substrate of dielectric function $\epsilon_{0}(\omega)$, a straightforward calculation yields the following formula: ${ }^{24}$

$\xi_{0}(\mathbf{q}, \omega)=\xi_{0}(q, \omega)=\epsilon_{1} \operatorname{coth} q d-\frac{\left(\epsilon_{1} / \sinh q d\right)^{2}}{\epsilon_{1} \operatorname{coth} q d+\epsilon_{0}}$.

The dielectric response of the magnesium substrate $\epsilon_{0}$ is modeled by introducing a Drude contribution of the form

$$
\epsilon_{0}(\omega)=\frac{-\omega_{p}^{2}}{\omega^{2}+i \gamma \omega \omega_{p}}
$$

The surface-plasmon frequency $\omega_{p}=7.2 \mathrm{eV}$ was measured by Ghijsen et al. ${ }^{25}$ and the damping ratio $\gamma=0.2$ was deduced from the width of the loss peak. To describe the dielectric function of the oxygen layer $\left(\epsilon_{1}\right)$ we take the optical constants measured by HREELS on MgO (see Sec. III B 1).

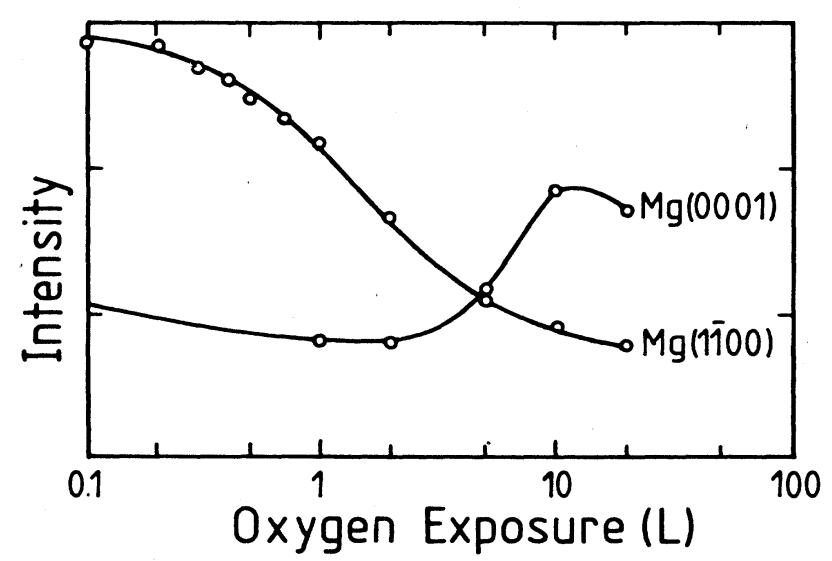

FIG. 8. Intensity variation of the elastic peaks recorded during oxygen exposure of $\mathrm{Mg}(0001)$ and $\mathrm{Mg}(1 \overline{1} 00)$. 
For vanishingly small oxide thickness $(d \rightarrow 0)$ the response function of the system $\operatorname{Im}\left\{-1 /\left[\xi_{0}(q, \omega)+1\right]\right\}$ has two poles located at the LO $\left(716 \mathrm{~cm}^{-1}\right)$ and TO $(393$ $\mathrm{cm}^{-1}$ ) frequencies of $\mathrm{MgO}$, respectively. From an inspection of the boundary conditions used to compute the classical energy-loss probability, it appears that the LO frequency is associated with a surface-phonon mode of $\mathrm{MgO}$ type and that the TO frequency must be attributed to a mode localized at the interface between the metal and the oxide layer (interface mode). The intensity of the interface mode is very low in comparison to the surface mode, because it is heavily screened by the metal electrons. With increased oxide thickness, the surfacephonon frequency shifts to lower values and tends asymptotically to the Fuchs-Kliewer phonon frequency of $\mathrm{MgO}$ (Fig. 9). On the other hand, the interface phonon frequency increases with layer thickness, but its intensity drops very rapidly and it becomes undetectable after a few angstroms oxide thickness.

One can of course question the applicability of a macroscopic continuum theory such as the dielectric theory to the description of very thin oxide layers for which quantum effects should play an important role. Nevertheless, it is interesting to see that two frequency modes are obtained theoretically and observed experimentally in the early stages of oxidation. Unfortunately, the comparison between theory and experiment ends here because, contrary to the predicted decrease in frequency, an increase is observed for peak $P 2$ (surface phonon) which starts at the very low value of $640 \mathrm{~cm}^{-1}$ and overrides the Fuchs-Kliewer mode at higher oxygen exposure. It is clear that the present situation is to be very different from the case of an epitaxial dielectric layer supported on an isotropic substrate, e.g., epitaxial $\mathrm{CaF}_{2}$ on $\mathrm{Si}(111),{ }^{8}$ where dielectric theory is applicable in the intermediate thickness range of $100 \AA$.

If peak $P 2$ is attributed to the surface phonon frequency of the $\mathrm{MgO}$ layer, an interpretation other than the interface phonon must be found for peak $P 1$. The rapid intensity saturation of this peak suggests that it could be as-

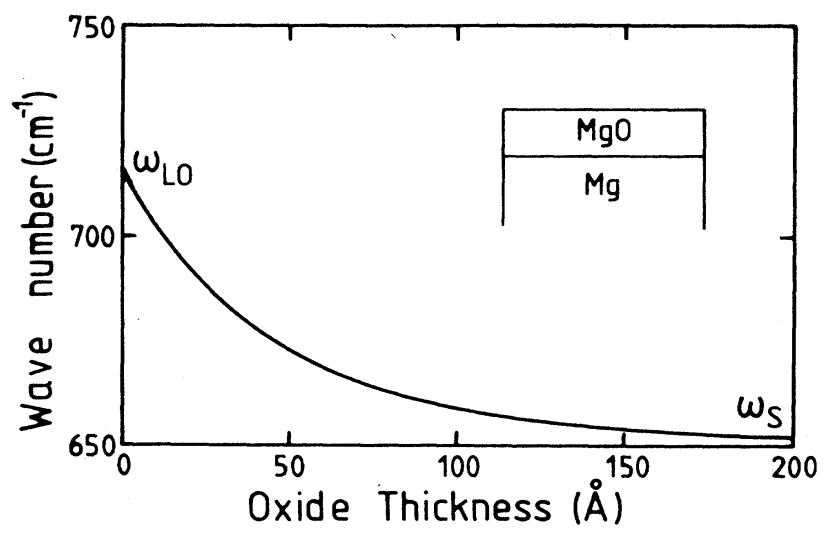

FIG. 9. Theoretical behavior of the optical surface-phonon frequency vs oxide thickness, calculated from the dielectric theory in the case of a thin crystalline $\mathrm{MgO}$ layer supported on magnesium. sociated with chemisorbed oxygen at the surface. Hydroxyl vibrations can be ruled out, because the corresponding $\mathrm{O}-\mathrm{H}$ stretching band $\left(3700 \mathrm{~cm}^{-1}\right)$ is not detected below $10 \mathrm{~L}$ although peak $P 1$ is present from the start.

\section{Discussion}

We first concentrate our discussion on the interpretation of the second peak $P 2$. Three pieces of evidence demonstrate that $P 2$ is the vibrational fingerprint of magnesium-oxide formation at the surface: (1) its frequency is always close to the frequency of the FuchsKliewer phonon of $\mathrm{MgO}$, (2) its presence from the beginning, and continued increase in intensity, is consistent with the scheme of immediate oxide formation deduced from other spectroscopies, and (3) the observation of multiple energy-loss peaks and energy-gain peaks attests to its "phonon" character, which is also confirmed by the observation of epitaxial $\mathrm{MgO}(111)$ domain formation reported from LEED analysis. ${ }^{1,3,4} P 2$ is thus attributed to a Fuchs-Kliewer optical surface mode of the $\mathrm{MgO}$ layer. We then have to interpret its frequency shift observed during early oxidation.

Usually, coverage-dependent frequency shifts measured in an adsorbate layer are interpreted as resulting from a "physical" and a "chemical" shift. ${ }^{26}$ The former is related to lateral interactions between the oscillating dipoles. The effect is a depolarization of the dynamical dipole increasing the force constant and thus inducing a shift to higher frequencies. The chemical shift is due to interactions with the substrate. both contributions can be separated if the experiments are carried out with different isotopic mixtures.

In the present context, these frequency shifts are ignored since the interaction of oxygen with magnesium single-crystal surfaces results in the immediate formation of oxide by oxygen incorporation. The oxygen molecule is immediately dissociated (either by its contact with the magnesium surface or by the hot ion gauge filament) and buried under the surface where it reacts to form magnesium oxide. This process of direct incorporation is confirmed by XPS results and by all the work-function measurements. The observed frequency shift can then only be due to the change of the interatomic $\mathrm{Mg}-\mathrm{O}$ distance. This interpretation is confirmed by a geometrical model, based on the comparison between the lattices of the metal and its oxide.

The position of the oxygen atoms in the Mg lattice can be easily deduced if one compares the fcc $\mathrm{MgO}$ lattice with the hcp Mg lattice. The resulting chemisorption site is an octahedral site where the oxygen atom has an environment quite similar to the one it has in magnesium oxide, except that the $\mathrm{Mg}-\mathrm{O}$ distance is $2.27 \AA$ instead of $2.10 \AA$ in $\mathrm{MgO}$. The oxygen atoms located in these sites are regularly distributed in planes parallel to $\mathrm{Mg}(0001)$ and exhibit the same symmetry as the $\mathrm{Mg}$ basal plane.

The fact that the chemical bonding of the oxide is completed at once suggests an extension of the BirgeMecke (BM) rule (usually applied to diatomic molecules in the gas phase ${ }^{27}$ ) to describe the frequency shift quantitatively. The empirical BM law relates the vibrational 
frequency $(\omega)$ of a molecule to its interatomic distance $(r):$

$$
r^{2} \omega=\text { const . }
$$

Taking for the incipient oxidation stage the observed frequency of $620 \mathrm{~cm}^{-1}$ and the "unrelaxed" $\mathrm{Mg}-\mathrm{O}$ distance of $2.27 \AA$, it is possible to calculate the constant in Eq. (7) and to plot the function (Fig. 10). We can then predict that the peak frequency measured at $660 \mathrm{~cm}^{-1}$ at saturation $(1000 \mathrm{~L})$ corresponds to an interatomic distance of $2.20 \AA$, i.e., an oxygen-induced lattice contraction of $3 \%$. Moreover, if one assumes a complete reconstruction of the $\mathrm{Mg}+\mathrm{O}$ lattice into a perfect $\mathrm{MgO}$ lattice $(\mathrm{Mg}-\mathrm{O}$ bond $=2.10 \AA)$ the $\mathrm{BM}$ law extrapolates a phonon frequency of $724 \mathrm{~cm}^{-1}$. This value is close to the LO-phonon frequency of $\mathrm{MgO}$ as deduced from HREELS measurements $\left(716 \mathrm{~cm}^{-1}\right)$ applying the Lyddane-Sachs-Teller relation. We emphasize that the LO frequency was predicted by dielectric theory for a very thin film of $\mathrm{MgO}$ supported on the metal. The correspondence of this frequency $\left(716 \mathrm{~cm}^{-1}\right)$ with the frequency deduced from the BM relation $\left(724 \mathrm{~cm}^{-1}\right)$ confirms the proposed oxidation model. The initial fluctuations of frequency can be related to the buildup of the first oxide layer, thus indicating monolayer formation at $4 \mathrm{~L}$ for $\mathrm{Mg}(0001)$ and at $2 \mathrm{~L}$ for $\mathrm{Mg}(1 \overline{100})$. It is only after the first oxide layer is complete that one observes a lattice contraction during further oxidation.

We can try to calibrate the oxygen uptake using the dipolar theory. In a famous paper on the quantummechanical treatment of HREELS, Evans and Mills ${ }^{28}$ proposed a formula for the relative intensity of an energy-loss peak related to phonon excitation in a layer of adsorbed dipoles:

$$
\begin{aligned}
\frac{I_{1}}{I_{0}}= & \frac{\hbar}{4 \pi \epsilon_{0} a_{0}} \frac{n_{0} e^{* 2}}{\mu}\left[\frac{\epsilon}{\epsilon+1}\right]^{2} \frac{F\left(E_{0}, \psi_{c}, \theta, \omega_{s}\right)}{E_{0} \cos \theta} \\
& \times \frac{n_{s}\left(\hbar \omega_{s}\right)+1}{\omega_{s}} .
\end{aligned}
$$

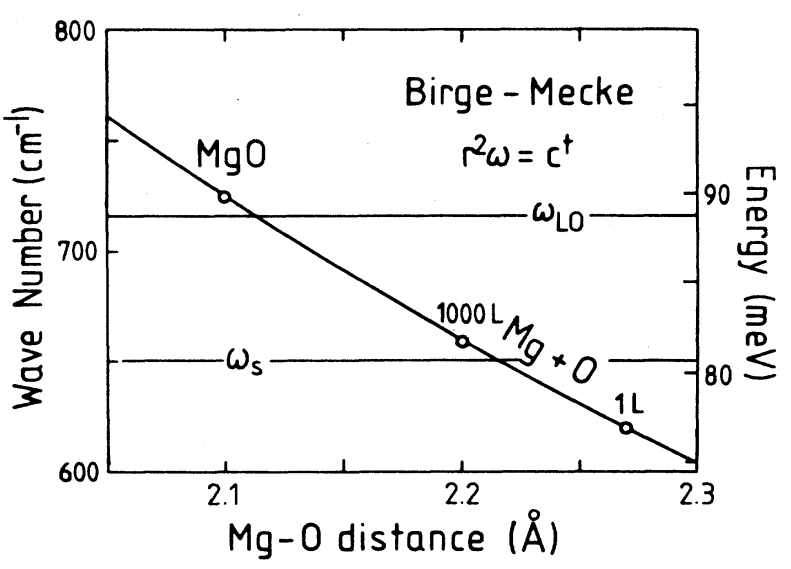

FIG. 10. Birge-Mecke relation as computed from the HREEL spectra at $0.5 \mathrm{~L}$ oxygen. The point labeled $\mathrm{MgO}$ extrapolates a full reconstruction of the $\mathrm{MgO}$ lattice (see text). $\omega_{s}=\omega_{\mathrm{FK}}$ (FK denotes Fuchs-Kliewer) is the frequency of the optical surface phonon measured on $\mathrm{MgO}(001)$ and $\omega_{\mathrm{LO}}$ is the longitudinal-optical phonon of $\mathrm{MgO}$. $a_{0}$ is the Bohr radius, $\epsilon_{0}$ is the vacuum permittivity, $n_{0}$ is the adsorbate concentration on the surface, $e^{*}$ is the effective charge associated with the dipolar momentum of the vibration, $\mu$ is the reduced mass of the oscillator, $\theta$ is the angle of incidence, $E_{0}$ is the incident electron energy, $\epsilon$ is the substrate dielectric constant, $\psi_{c}$ is half of the analyzer angle of acceptance, $n_{s}$ is the Bose-Einstein distribution, and $\omega_{s}$ is the phonon frequency. The amplitude factor $F$ is given by

$$
\begin{aligned}
F\left(E_{0}, \psi_{c}, \theta, \omega_{s}\right)= & \frac{\sin ^{2} \theta-2 \cos ^{2} \theta}{\left[\frac{\hbar \omega_{s}}{2 \psi_{c} E_{0}}\right)^{2}+1} \\
& +\left(1+\cos ^{2} \theta\right) \ln \left[1+\left(\frac{2 \psi_{c} E_{0}}{\hbar \omega_{s}}\right)^{2}\right] .
\end{aligned}
$$

If Eq. (8) applies in our case, we should find a crosssection dependence of $E_{0}^{-1}$ instead of $E_{0}^{-1 / 2}$ as previously found for the Fuchs-Kliewer phonon of $\mathrm{MgO}$. Therefore, a systematic investigation of the relative intensities versus impact energy was carried out from 5 to $100 \mathrm{eV}$. An exposure of $10 \mathrm{~L}$ was chosen because at lower coverages the peak intensity was not constant for a long enough time due to continued oxidation from the ambient oxygen. The result, plotted in Fig. 11, clearly indicates an $E_{0}^{-1}$ dependence in agreement with Eq. (8).

Equation (8) was then numerically evaluated with $[\epsilon /(\epsilon+1)]^{2}=1$, which is justified for a metal in this frequency range. Setting $e^{*}$ to the Szigeti charge of $\mathrm{MgO}$ $(0.83 e)$ as deduced from our HREELS measurements on $\mathrm{MgO}$ single crystals, one obtains

$$
I_{2} / I_{0}=0.034 n_{0} \text {, }
$$

where $n_{0}$ is expressed in monolayer units for $\mathrm{Mg}(0001)$. From the relative intensities shown in Fig. 7, a monolayer

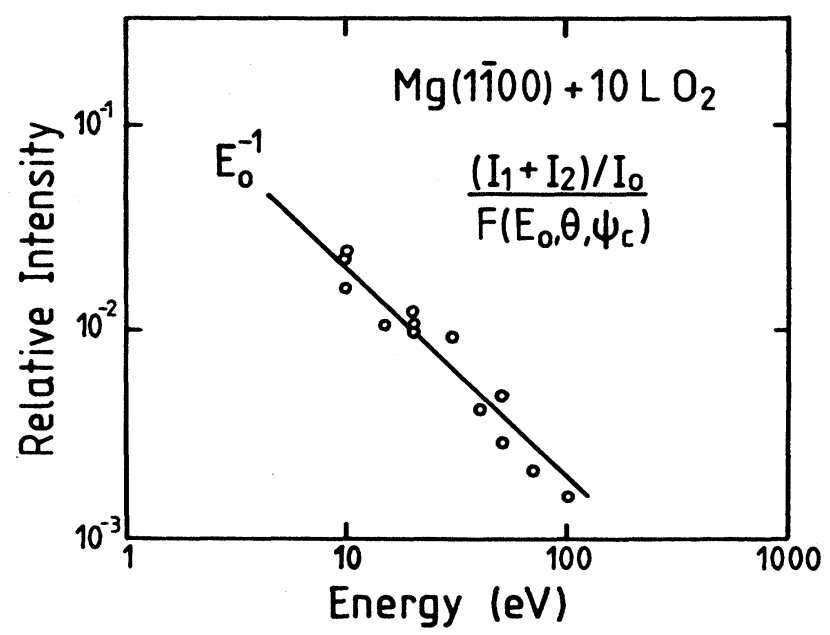

FIG. 11. Electron impact energy dependence of the HREEL reduced cross section of peaks $P 1$ and $P 2$ measured after $10 \mathrm{~L}$ exposure on $\mathrm{Mg}(1 \overline{1} 00)$. Data reduction is performed by dividing the peak intensities by the amplitude factor $F$ described in the text [Eq. (9)] in order to get the $E^{-1}$ law. 
of surface oxide is formed on $\mathrm{Mg}(0001)$ for an oxygen exposure of $4 \mathrm{~L}$ which is close to the inflection point and confirms the qualitative evaluation based on the inspection of the frequency shifts.

For the $\mathrm{Mg}(1 \overline{100})$ surface, the equivalent formula is

$$
I_{2} / I_{0}=0.030 n_{0} \text {. }
$$

A monolayer of oxide is obtained after an exposure of 2 $\mathbf{L}$, i.e., twice as fast as on $\mathrm{Mg}(0001)$. The interaction of oxygen with the more open $\mathrm{Mg}(1 \overline{1} 00)$ surface destroys its symmetry as confirmed by the decrease of the elastic intensity (Fig. 8) and by the LEED measurements of Hayden et al. ${ }^{3}$ The loss of symmetry enhances the oxidation process. For the $\mathrm{Mg}(0001)$ surface the increase in the elastic intensity and the persistence of the primitive $(1 \times 1)$ LEED pattern up to $6 \mathrm{~L}$ are in keeping with ordered adsorption at the octahedral sites described previously.

We now come to the interpretation of peak $P 1$, which is observed at a lower frequency than $P 2$. One must keep in mind that at low exposure $P 1$ and $P 2$ behave very similarly, and that the work-function results reported by other authors ${ }^{1,3}$ indicate adsorption sites under the surface. We propose two interpretations.

(1) Chemisorbed oxygen: There is a tetrahedral site on the $\mathrm{Mg}$ surface which could accommodate one oxygen atom during the early stage of oxidation, and that would be filled preferentially in the neighborhood of the oxide islands.

(2) After completion of the first oxide (and chemisorbed) monolayer, $P 1$ could also be the signature of interstitial oxygen, diffusing through the oxide barrier to carry the oxidation further inside the metal.

The second interpretation is confirmed by the spectra in Fig. 12 which have been recorded on a nonannealed

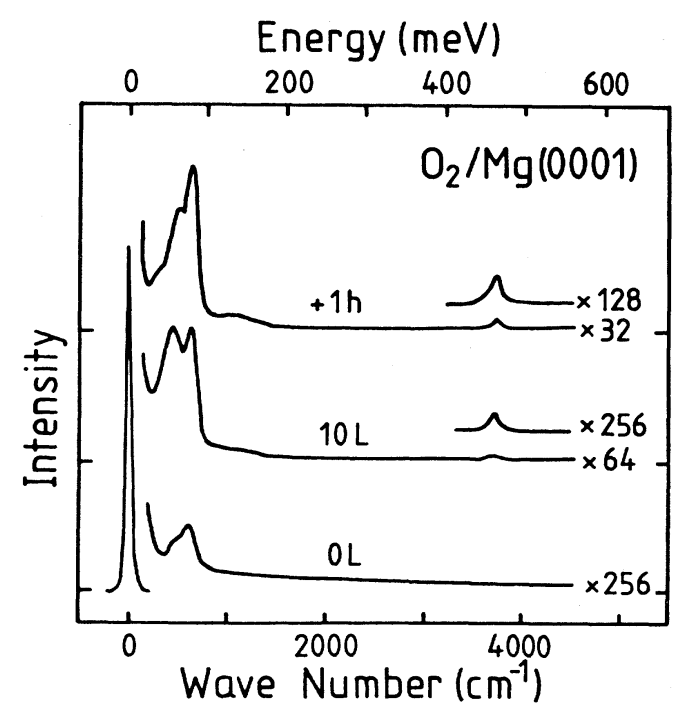

FIG. 12. Energy-loss spectra recorded after specular reflection of $5.5-\mathrm{eV}$ electrons on a rough (nonannealed) $\mathrm{Mg}(0001)$ surface. The first spectrum is taken before oxygen exposure, the second one after $10 \mathrm{~L} \mathrm{O}_{2}$ exposure, and the third one $1 \mathrm{~h}$ later without subsequent oxygen exposure.
$\operatorname{Mg}(0001)$ sample. The residual roughness favors the formation of interstitial oxygen ${ }^{1}$ which is then automatically converted into oxide as shown by the third spectrum taken one hour later without further exposure to oxygen.

From a detailed inspection of the shape and width of the peaks it is clear that several components contribute to $P 1$ after monolayer completion (i.e., $>10 \mathrm{~L}$ ). These components cannot be resolved with the present energy resolution but they are thought to result from the two oxygen species and from the vibrations of $\mathrm{OH}$ species, which are identified by their stretching vibrations at $3700 \mathrm{~cm}^{-1}$. Another conclusion is that $\mathrm{OH}$ chemisorption is observed only after the buildup of the first oxide monolayer, indicating that $\mathrm{OH}$ chemisorbs on magnesium oxide and not directly on the clean metal.

\section{E. Conclusions and comparison with other spectroscopies}

From the HREELS results, two different atomic oxygen species are observed during early oxidation of crystalline magnesium surfaces: surface oxide and chemisorbed oxygen. The evolution of the oxide could be quantitatively described by dipolar theory. Chemisorbed oxygen is present from the beginning and seems to accompany the oxide formation up to the completion of the first monolayer. The evolution of both species is similar for the two surfaces $\mathrm{Mg}(0001)$ and $\mathrm{Mg}(1 \overline{100)}$ but the latter is more reactive. We now briefly compare our results with those obtained from other spectroscopies.

Work-function measurements indicate an initial decrease during oxygen exposure of the two faces. This result has been used to determine possible oxygen sites, since it restricts the sites where oxygen can be incorporated. The slight increase observed after $10 \mathrm{~L}$ could be due to the presence of hydroxyl radicals adsorbed on the surface.

LEED measurements have been reported by Namba et al., ${ }^{1}$ Hayden et al. ${ }^{3}$ and Flodström and Martinsson. ${ }^{4}$ They agree with the octahedral site for the surface oxide, and confirm the coverage for the formation of the first oxide monolayer and the different reactivities of the two surfaces $\mathrm{Mg}(0001)$ and $\mathrm{Mg}(1 \overline{1} 00)$.

EEL (medium resolution) and Auger spectroscopies ${ }^{1,3,4}$ cannot distinguish the different oxygen species and their results can only be globally compared to the HREELS results. However, they do not even find a different reactivity for the two surfaces $\mathrm{Mg}(0001)$ and $\mathrm{Mg}(1 \overline{1} 00)$.

The clearest evidence for the presence of two oxygen species during early $\mathrm{Mg}$ single-crystal oxidation has been reported by Ghijsen et al. using XPS. ${ }^{2}$ They detected two oxygen species with different $\mathrm{O} 1 s$ core-level binding energies. It is clear that one species must be attributed to our surface oxide peak $P 2$. They tentatively assigned the other species to either interstitial oxygen or defective oxide because it appeared only after the "true" oxide. On the basis of the XPS results alone it would be difficult to rule out the presence of hydroxyl groups which would have similar binding energies. It was suggested that more information could be obtained from a detailed inspection of the $\mathrm{Mg} 2 p$ core-level peaks measured after low oxygen exposure. In order to get the best energy resolution and 
surface sensitivity, the photoemission measurements described in the following section were carried out with synchrotron radiation.

\section{PHOTOEMISSION RESULTS}

\section{A. Experimental details}

The photoemission experiments were performed on the Flipper-II beamline at the Hamburg synchrotronradiation laboratory (HASYLAB, DESY Hamburg, FRG). A detailed description of the experimental setup can be found elsewhere. ${ }^{29}$ The sample used for these measurements was oriented in the (1100) direction and was cleaned by ion bombardment without subsequent annealing. Diffuse LEED $(1 \times 1)$ patterns were observed on the clean surface which was presumably more reactive due to the roughness resulting from the ion sputtering. Oxygen exposures were performed in an introduction chamber adjacent to the preparation chamber of the spectrometer. Oxygen pressures of $5 \times 10^{-7} \mathrm{~Pa}$ were used and the residual pressure of the system was $5 \times 10^{-9} \mathrm{~Pa}$.

\section{B. Results}

Electron energy-distribution curves (EDC's) from the $\mathrm{Mg} 2 p$ core level and from the valence band were measured before and after the oxygen exposure. The $\mathrm{Mg} 2 p$ results are shown in Fig. 13. A photon energy of $100 \mathrm{eV}$ was chosen for high surface sensitivity. Data analysis was performed following a procedure described elsewhere. $^{30}$ After suitable background subtraction, spinorbit doublets of Doniach-Šunjić line shape ${ }^{31}$ were fitted to the spectra. On the clean surface, two doublets were needed: one surface and one bulk component. The optimized values of the parameters agree with those reported by Kammerer et al. ${ }^{32}$ They are summarized in Table II. The bulk $\mathrm{Mg} 2 p_{3 / 2}$ binding energy was measured as $49.74 \pm 0.05 \mathrm{eV}$ below the Fermi level. A surface shift of $0.19 \mathrm{eV}$ was deduced. Satellites corresponding to bulkand surface-plasmon excitation were observed at $\hbar \omega_{p}=11.0 \mathrm{eV}$ and $\hbar \omega_{s}=7.3 \mathrm{eV}$. With increasing oxygen exposure, one broad structure develops on the highbinding-energy side of the $\mathrm{Mg} 2 p$ peak. Starting as a shoulder, it grows to become equal in height to the $\mathrm{Mg} 2 p$ peak after $2 \mathrm{~L}$ oxygen exposure, corresponding to one monolayer (ML) coverage as estimated by HREELS. After $3 \mathrm{~L}$, the oxide $\mathrm{Mg} 2 p$ peak dominates the spectrum and the metallic $\mathrm{Mg} 2 p$ is observed as a shoulder.

The EDC's from the valence band before and after oxygen exposure were recorded with a photon energy of 30 $\mathrm{eV}$. This gave the highest photoemission cross section for the $\mathrm{O} 2 p$ level and a high surface sensitivity. The cleanest spectrum still exhibited a small amount of oxygen-related structure around $6 \mathrm{eV}$ binding energy, but the sharp metallic Mg Fermi edge indicated a low level of oxygen contamination. With increasing oxygen exposure, the $\mathrm{O} 2 p$ peak at $6.0 \mathrm{eV}$ grows rapidly, together with another structure seen as a shoulder on the high-binding-energy side.

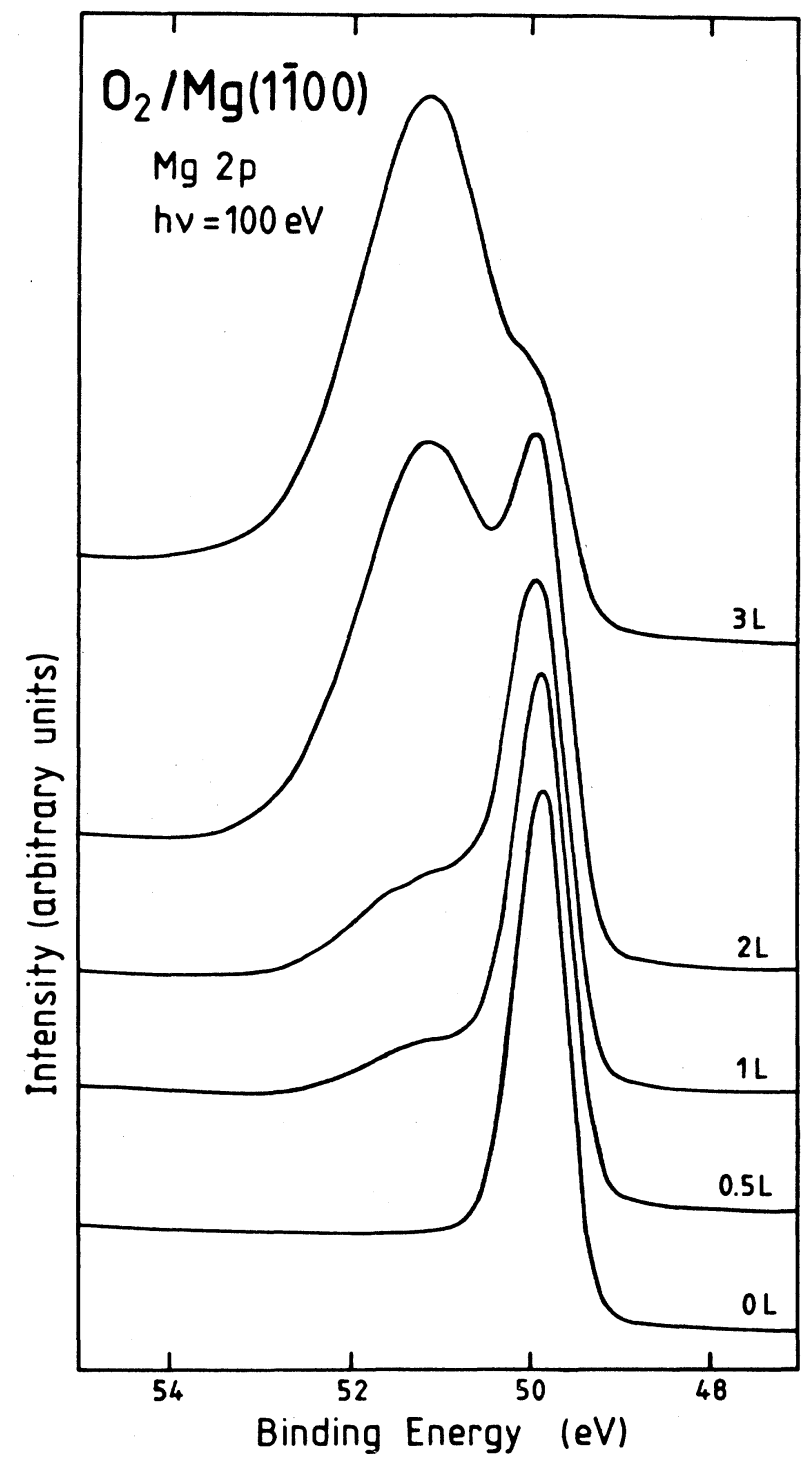

FIG. 13. $\mathrm{Mg} 2 p$ core-level energy-distribution curves from $\mathrm{Mg}(1 \overline{1} 00)$ after different oxygen exposures. The photon energy is $100 \mathrm{eV}$. The spectra have been normalized to the same excitation intensity.

\section{Discussion}

Previously, only one broad ( $1-2 \mathrm{eV}) \mathrm{Mg} 2 p$ oxide component has been observed in photoemission studies on the oxidation of polycrystalline or single-crystal magnesium

TABLE II. $\mathrm{Mg} 2 p_{3 / 2}$ core-level binding energies $E_{B}(\mathrm{eV})$, width (meV), and singularity index $\alpha$ for the surface and bulk components in the Mg $2 p$ EDC's. A Gaussian broadening of $330 \mathrm{meV}$ was used for the fit. The spin-orbit splitting is $\Delta_{\text {s.o. }}=250 \mathrm{meV}$ and the intensity ratio is 0.5 .

\begin{tabular}{lccc}
\hline \hline & $E_{B}(\mathrm{eV})$ & FWHM $(\mathrm{meV})$ & $\alpha$ \\
\hline Mg 2p bulk & 49.74 & 68 & 0.129 \\
$\mathrm{Mg} \mathrm{2p}$ surface & 49.93 & 94 & 0.122 \\
\hline
\end{tabular}


using XPS (Ref. 2) or synchrotron radiation. ${ }^{32}$ The broad oxide component is shifted by about $1.3 \mathrm{eV}$ to higher binding energy, with respect to the metal line. ${ }^{33,34} \mathrm{We}$ were unable to fit our spectra with only one oxide species and found it necessary to use at least two components as is shown in Fig. 14 for the spectrum recorded after $1 \mathrm{~L}$ exposure. Hereafter, these two oxygen-related doublets will be referred to as lower-binding-energy (LBE) and higher-binding-energy (HBE) peaks, respectively.

Upon oxygen adsorption the $\mathrm{Mg} 2 p$ surface and bulk components are attenuated but the $\mathrm{Mg} 2 p$ surface to $\mathrm{Mg}$ $2 p$ bulk intensity ratio is almost unaffected. At the same time, the binding-energy difference between the components increases from 190 to $460 \mathrm{meV}$ and a slight increase of the FWHM from 94 to $125 \mathrm{meV}$ is observed. We explain these changes by a progressive replacement of the $\mathrm{Mg} 2 p$ surface component by a contribution coming from the interface between the bulk $\mathbf{M g}$ and the $\mathbf{M g O}$ layer. The thermodynamical model used by Kammerer et $a l .{ }^{32}$ to explain the surface shift for clean $\mathrm{Mg}$ obviously has to be modified to allow for the presence of an interface. Moreover, the final-state screening is also expected to change due to the presence of the MgO layer. However, the quantitative determination of these combined effects is beyond the scope of this work.

We now focus our attention on the identification of the two oxygen-related components HBE and LBE. As LBE corresponds to the peak position observed on heavily oxidized magnesium, it can be identified as the $\mathrm{MgO}$ species. Its chemical shift of $1.08 \pm 0.15 \mathrm{eV}$ with respect to bulk metallic $\mathrm{Mg}$ is within the range of $0.8-1.3 \mathrm{eV}$ reported in the literature for $\mathrm{MgO} .^{34,35}$ We achieve better accuracy because we are working with single crystals and separate

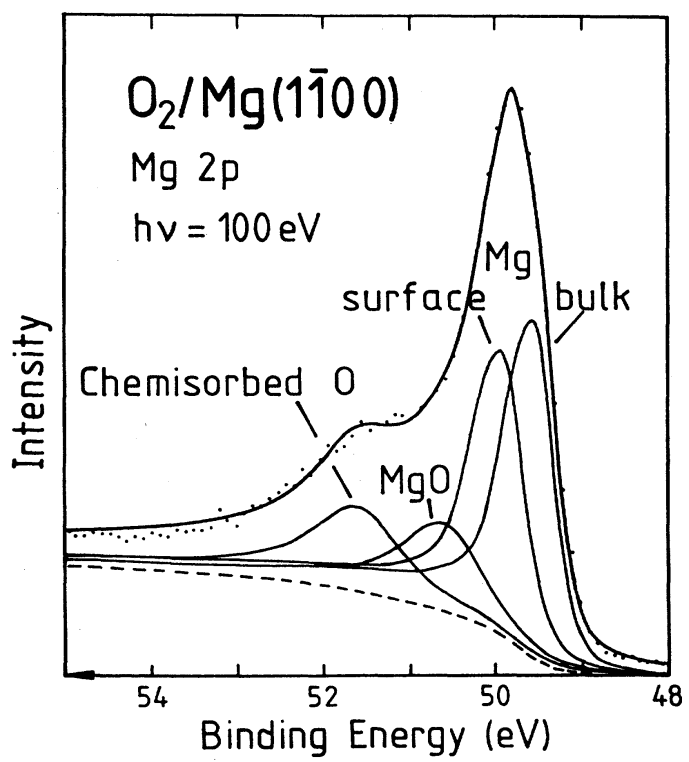

FIG. 14. Curve-fitting results to the $\mathrm{Mg} 2 p$ core-level EDC recorded on $\mathrm{Mg}(1 \overline{1} 00)$ after $1 \mathrm{~L} \mathrm{O}_{2}$ exposure. The excitation energy is $100 \mathrm{eV}$. The dashed line represents the background. The four individual spin-orbit doublets discussed in the text, as well as their sum, are shown. the bulk and surface contributions. Almost all other published results were obtained on polycrystalline samples, except the work by Ghijsen et al. on $\mathrm{Mg}(0001),{ }^{2}$ who obtained a chemical shift of $1.05 \mathrm{eV}$ assuming only one oxide species and one metallic component to be present.

The second doublet (HBE) at a binding energy of $51.44 \pm 0.10 \mathrm{eV}$ is attributed to oxygen chemisorbed at the surface of the oxide layer in formation. Consequently, it should correspond to the $P 1$ species observed by HREELS. Its smaller binding-energy shift $(0.65 \pm 0.15 \mathrm{eV}$ instead of $1.08 \pm 0.15 \mathrm{eV}$ ) indicates an incomplete charge transfer which is the cause of its lower vibrational frequency. The intensity ratio HBE:LBE is almost equal to unity, independent of the oxygen exposure. This is in keeping with the HREELS results where $P 1$ and $P 2$ grow simultaneously from 0 to $10 \mathrm{~L} \mathrm{O}_{2}$ exposure. Table III summarizes the mean values of the parameters used to fit the spectra. A quantitative analysis of the peak intensities is not relevant here due to their lack of reproducibility which was probably due to the increasing roughness of the surface with successive sputtering cycles. The important result of this section is the confirmation of the existence of two oxygen species as observed by HREELS during early exposure to oxygen and their identification as $\mathrm{MgO}$ and oxygen chemisorbed on the oxide layer, respectively.

The presence of two oxygen species during oxidation of $\mathrm{Mg}(0001)$ had already been deduced by Ghijsen et al. ${ }^{2}$ from an inspection of the O $1 s$ peaks recorded with XPS. Their conclusions were that one peak at about $530 \mathrm{eV}$ binding energy should be attributed to the oxygen of magnesium oxide and that the other one at $533 \mathrm{eV}$ could be due to defective oxide or chemisorbed oxygen on top of the oxidized substrate. This second $\mathrm{O} 1 s$ species seems to correspond to our $\mathrm{HBE}$ peak but there is a difference in that it only appeared after the development of the oxide. The probable explanation for this discrepancy lies in the different energy resolution and surface sensitivities of the two techniques.

\section{CONCLUSIONS}

The high sensitivity of HREELS enabled us to identify the simultaneous ${ }^{36}$ appearance of two oxygen species during early oxidation of magnesium single-crystal surfaces. Referring to the results obtained on $\mathrm{MgO}$, it was possible to quantitatively describe the oxidation process by assuming an adsorbate-induced lattice contraction. However,

TABLE III. Average values of the relevant parameters used to fit the spectra in the range of $0.5-3 \mathrm{ML}$ oxygen exposure.

\begin{tabular}{llcc}
\hline \hline & \multicolumn{1}{c}{$\begin{array}{c}E_{B} \\
(\mathrm{eV})\end{array}$} & $\begin{array}{c}\text { FWHM } \\
(\mathrm{meV})\end{array}$ & $\alpha$ \\
\hline Mg 2p bulk & $49.71 \pm 0.05$ & $68 \pm 8$ & $0.132 \pm 0.003$ \\
Mg $2 p$ surface & $49.93-50.20^{\mathrm{a}}$ & $94-125^{\mathrm{a}}$ & $0.122-0.132^{\mathrm{a}}$ \\
LBE & $50.79 \pm 0.10$ & $308 \pm 30$ & $<0.1$ \\
HBE & $51.44 \pm 0.10$ & $380 \pm 20$ & $<0.1$ \\
\hline \hline
\end{tabular}

${ }^{\text {a }}$ Depending on the oxygen exposure. 
although this reconstruction is plausible we admit that the HREELS data only provide indirect evidence. It would be interesting to confirm this working by measuring the $\mathrm{Mg}-\mathrm{O}$ bond length during oxygen exposure with extended $\mathrm{x}$-ray-absorption fine-structure spectroscopy or to calculate the $\mathrm{Mg}+\mathrm{O}$ lattice relaxation from the analysis of LEED $I-V$ curves. The second oxygen species is attributed to oxygen chemisorbed on the oxide being formed and could only be interpreted qualitatively. The HREELS observations are confirmed by the analysis of the $\mathrm{Mg} 2 p$ EDC's recorded using synchrotron radiation. The results presented here do not indicate the occurrence of a "precursor"-type oxygen species before oxide formation.

\section{ACKNOWLEDGMENTS}

We are grateful to $\mathrm{Ph}$. Lambin, J. P. Vigneron, and $\mathrm{A}$. A. Lucas for their theoretical support and to M. Liehr for his participation in the $\mathrm{MgO}$ measurements. This work is supported by the Belgian Fund for Joint Basic Research (FJBR), National Fund for Scientific Research (NFSR), and Ministry for Science Policy (IRIS project). For the synchrotron-radiation measurements performed at HASYLAB we also acknowledge the support of the German Bundesministerium für Forschung und Technologie (BMFT) under Project Nos. 05390 CAB and 05250 CA. One of us (J.J.P.) is grateful to the NFSR for financial support.
*Present address: Technical Physics Laboratory, University of Groningen, Nijenborgh 18, NL-9747 AG Groningen, The Netherlands.

${ }^{1}$ H. Namba, J. Darville, and J. M. Gilles, Surf. Sci. 108, 446 (1981).

${ }^{2}$ J. Ghijsen, H. Namba, P. A. Thiry, J. J. Pireaux, and R. Caudano, Appl. Surf. Sci. 8, 387 (1981).

${ }^{3}$ B. Hayden, E. Schweizer, R. Kötz, and A. M. Bradshaw, Surf. Sci. 111, 26 (1981). R. Kötz, B. Hayden, E. Schweizer, and A. M. Bradshaw, Surf. Sci. 112, 229 (1981).

${ }^{4}$ S. A. Flodström and C. W. B. Martinsson, Surf. Sci. 118, 513 (1982).

${ }^{5}$ M. Liehr, P. A. Thiry, J. J. Pireaux, and R. Caudano, Phys. Rev. B 33, 5682 (1986).

${ }^{6}$ P. A. Thiry, M. Liehr, J. J. Pireaux, and R. Caudano, Phys. Rev. B 29, 4824 (1984).

${ }^{7}$ Ph. Lambin, J. P. Vigneron, and A. A. Lucas, Phys. Rev. B 32, 8203 (1985).

${ }^{8}$ M. Liehr, P. A. Thiry, J. J. Pireaux, and R. Caudano, Phys. Rev. B 34, 7471 (1986).

${ }^{9}$ Ph. Lambin, J. P. Vigneron, A. A. Lucas, P. A. Thiry, M. Liehr, J. J. Pireaux, R. Caudano, and T. Kuech, Phys. Rev. Lett. 56, 1842 (1986).

${ }^{10}$ A. A. Lucas and M. Šunjić, Prog. Surf. Sci. 2, 75 (1972).

${ }^{11}$ See Refs. 7 and 10 and A. A. Lucas and J. P. Vigneron, Solid State Commun. 49, 327 (1984); Ph. Lambin, J. P. Vigneron, and A. A. Lucas, ibid. 54, 287 (1985).

${ }^{12}$ H. Ibach and D. L. Mills, Electron Energy Loss Spectroscopy and Surface Vibrations (Academic, New York, 1982).

${ }^{13}$ P. A. Thiry, M. Liehr, J. J. Pireaux, and R. Caudano, J. Electron Spectrosc. 39, 69 (1986).

${ }^{14}$ B. Piriou, C. R. Acad. Sci. 259, 1052 (1964); B. Piriou and F. Cabannes, ibid. 260, 841 (1965).

${ }^{15}$ J. R. Jasperse, A. Kahan, J. N. Plendl, and S. S. Mitra, Phys. Rev. 146, 526 (1966).

16J. J. Pireaux, P. A. Thiry, and R. Caudano, Surf. Sci. 162, 132 (1985).

${ }^{17}$ I. F. Chang and S. S. Mitra, Phys. Rev. B 5, 4094 (1972).

${ }^{18}$ M. Liehr, P. A. Thiry, J. J. Pireaux, and R. Caudano, J. Vac. Sci. Technol. A 2, 1079 (1984).

${ }^{19}$ H. Ibach, Phys. Rev. Lett. 24, 1416 (1970); in Festkörperprobleme (Advances in Solid State Physics), edited by P. Grosse (Pergamon/Vieweg, Braunschweig, 1971), Vol.
XI, p. 135.

${ }^{20} \mathrm{Ph}$. Lambin, A. A. Lucas, and J. P. Vigneron, Surf. Sci. 182, 567 (1987).

${ }^{21}$ P. A. Thiry, M. Liehr, J. J. Pireaux, and R. Caudano, Phys. Scr. 35, 368 (1987).

${ }^{22}$ Preliminary results have been presented in P. A. Thiry, J. Ghijsen, J. J. Pireaux, and R. Caudano, J. Electron Spectrosc. 29, 193 (1983).

${ }^{23}$ R. Sporken, P. A. Thiry, J. J. Pireaux, R. Caudano, and A. Adnot, Surf. Sci. 160, 443 (1985).

${ }^{24}$ A. A. Lucas, J. P. Vigneron, Ph. Lambin, P. A. Thiry, M. Liehr, J. J. Pireaux, and R. Caudano, Int. J. Quantum Chem., Quantum Chem. Symp. 19, 687 (1986).

25 J. Ghijsen, P. A. Thiry, J. J. Pireaux, and R. Caudano, Surf. Sci. 126, 177 (1983).

${ }^{26}$ A. M. Bradshaw, Appl. Surf. Sci. 11, 712 (1982).

${ }^{27}$ G. Herzberg, Molecular Spectra and Molecular Structure I. Spectra of Diatomic Molecules, 2nd ed. (Van Nostrand/ Reinhold, New York, 1950), p. 456.

${ }^{28}$ E. Evans and D. L. Mills, Phys. Rev. B 5, 4126 (1972).

${ }^{29}$ R. L. Johnson and J. Reichardt, Nucl. Instrum. Methods 208, 791 (1983).

${ }^{30}$ R. Sporken, P. A. Thiry, E. Petit, J. J. Pireaux, R. Caudano, J. Ghijsen, R. L. Johnson, and L. Ley, Phys. Rev. B 35, 7297 (1987).

${ }^{31}$ S. Doniach and M. Šunjić, J. Phys. C 3, 285 (1980).

${ }^{32}$ R. Kammerer, J. Barth, F. Gerken, C. Kunz, S. A. Flodström, and L. I. Johansson, Phys. Rev. B 26, 3491 (1982).

${ }^{33}$ S. A. Flodström, C. W. B. Martinsson, G. Kalkoffen, and C. Kunz, Mater. Sci. Eng. 42, 31 (1980).

${ }^{34}$ C. D. Wagner and P. Biloen, Surf. Sci. 35, 82 (1973).

${ }^{35}$ S. P. Kowalczyck, F. R. McFeely, L. Ley, V. T. Gritsyna, and D. A. Shirley, Solid State Commun. 23, 161 (1977).

${ }^{36}$ It must be pointed out that simultaneity refers here to the time scale of the above-described experiments, which is of the order of $10 \mathrm{~min}$. This might be too long to resolve in time the kinetics of the oxidation and chemisorption processes. Recently, some authors [see, e.g., W. Ho, J. Vac. Sci. Technol. A 3, 1432 (1985)] have reported new spectrometer improvements, allowing HREELS measurements to be taken on a millisecond scale. Such experiments would be very useful to demonstrate whether the two oxygen species are formed simultaneously or one after the other. 